\section{Diamo un posto al sole alla ricerca}
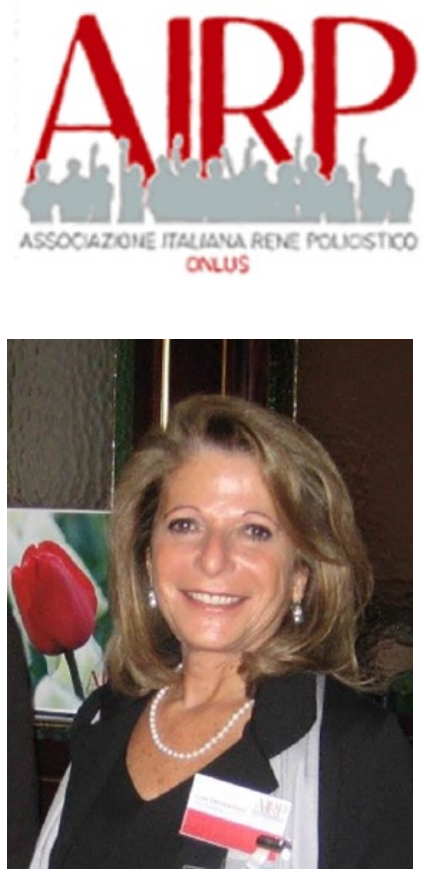

Carissimi Amici,

in questa occasione vorrei parlarvi di un argomento che per AIRP riveste un'importanza davvero fondamentale e che non abbiamo mai avuto l'occasione di approfondire insieme: il 5x1000.

Come sapete il nostro bilancio, che è pubblico e che trovate sul nostro sito web, deve al 5x1000 una notevole parte delle proprie entrate. Questo gettito, che anno per anno arriva grazie alla buona volontà di moltissimi nostri associati, oltre a diversi contributori esterni, è realmente la linfa vitale che ci consente di svolgere le molteplici attività della nostra associazione e che ci permette di finanziare importanti progetti di ricerca scientifica. Questa attività, lo sapete bene, è di gran lunga la parte più rilevante del nostro operato perché in essa sono riposte le speranze di poter migliorare le condizioni di vita dei nostri pazienti e di fare i passi necessari di un percorso che potrebbe portare ad una soluzione definitiva alla patologia del rene policistico.

Il 5x1000 è un'istituzione davvero importante ed è un segno di civiltà che caratterizza una società evoluta come quella in cui viviamo. Il suo ruolo non è solo significativo nel senso più concreto e fattuale della propria missione ma è anche un momento in cui il cittadino è chiamato a esercitare un proprio diritto democratico in totale autonomia, potendo liberamente scegliere a chi devolvere una quota del proprio carico fiscale, dirottandolo dallo Stato, che ne sarebbe il naturale destinatario, verso un Ente che intende sostenere.
Giornale di Tecniche Nefrologiche e Dialitiche 2018, Vol. 30(2) 122-123

(C) The Author(s) 2018

Article reuse guidelines:

sagepub.com/journals-permissions DOI: $10.1177 / 0394936218806859$

journals.sagepub.com/home/gtn

(\$SAGE
Del 5x1000 si parla tantissimo, specialmente nei mesi che precedono il momento in cui va fatta la dichiarazione dei redditi, ma non tutti hanno ben chiaro di cosa si tratta: proviamo a tracciarne le linee fondamentali.

Il $5 \times 1000$ è una misura fiscale che consente ai contribuenti di destinare una quota dell'IRPEF (pari, appunto, al 5 per mille dell'imposta sul reddito delle persone fisiche) a enti che si occupano di attività di interesse sociale, come associazioni di volontariato e di promozione sociale, Onlus, associazioni sportive che svolgono prevalentemente attività socialmente utili, enti di ricerca scientifica e sanitaria.

Non è una donazione, quindi non beneficia delle connesse agevolazioni fiscali, non si può detrarre dalle tasse, ma non comporta neppure oneri aggiuntivi in quanto il contribuente è comunque tenuto a pagare l'IRPEF. In pratica per il contribuente non costa nulla.

Istituito nel 2006 in forma sperimentale, il 5x1000 è diventato un mezzo di sostentamento indispensabile per gli enti non profit; grazie ad esso, i cittadini, con le loro preferenze, permettono uno sviluppo armonico e responsabile del cosiddetto terzo settore.

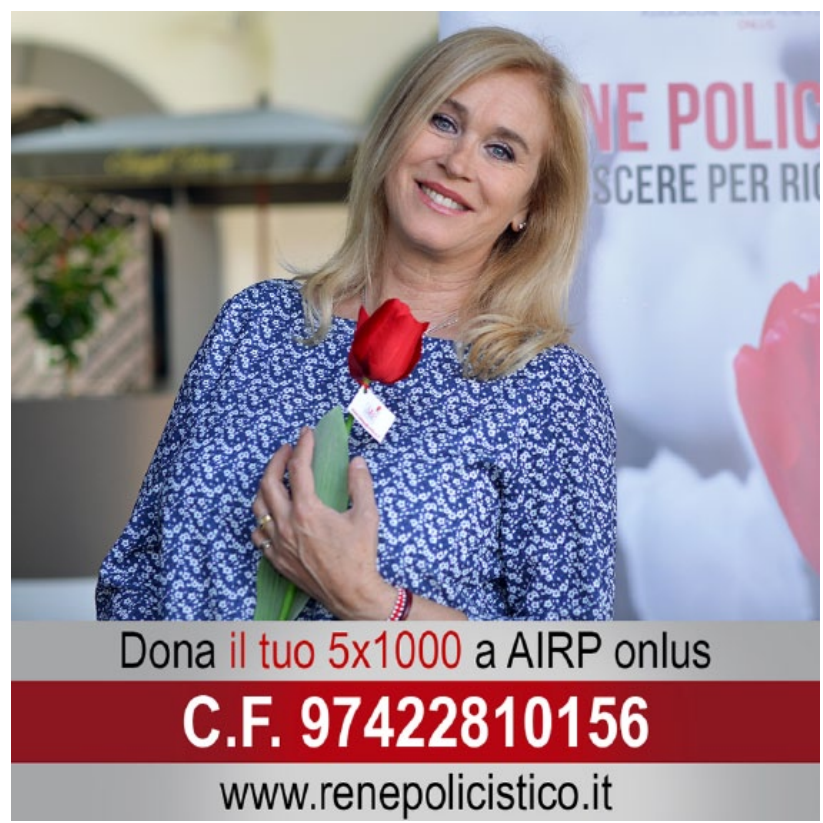


Il 5x1000 rappresenta per il cittadino un modo democratico per sostenere attività socialmente utili senza alcun aggravio. Potendo scegliere i beneficiari di una quota dell'imposta sul reddito, il contribuente esercita una sovra-nità inusuale, dal momento che di solito spetta al Parlamento stabilire l'impiego del gettito fiscale.

Il 5x1000 è una forma di sussidiarietà orizzontale, un principio che implica la collaborazione del cittadino con le istituzioni per l'attuazione di interventi socialmente rilevanti. Questo dà ad ognuno di noi il diritto/dovere di essere attori attivi e responsabili in questa scelta così importante.

Come avete avuto modo di osservare, capita di frequente che attori, sportivi e personaggi noti al grande pubblico offrano la propria collaborazione per diverse cause, proprio in concomitanza della richiesta del $5 \times 1000$ e quest'anno anche AIRP ha il proprio testimonial! Una delle protagoniste storiche di Un Posto Al Sole, da anni in testa tra le fiction più amate e seguite della televisione, è al nostro fianco: Marina Giulia Cavalli che nella fiction interpreta il ruolo di Ornella, una dottoressa molto impegnata tra ospedale e famiglia. Abbiamo incontrato Marina Giulia a Napoli, il luogo dove vengono realizzate le riprese di Un Posto $\mathrm{Al}$ Sole e con lei abbiamo realizzato le immagini per uno spot dedicato al 5x1000 dell'anno 2018. Voglio sentitamente ringraziare Marina Giulia Cavalli per aver così generosamente collaborato con noi dedicandoci tempo, passione ed energia e per averci donato la sua carismatica presenza per dare forza alle nostre istanze.

Un caro saluto a tutti voi.

Luisa Sternfeld Pavia

Un saluto e un abbraccio da parte mia e di tutto lo staff di AIRP, a tutti voi.

Luisa Sternfeld Pavia

Presidente AIRP

Presidente, AIRP - Associazione Italiana Rene Policistico Onlus

Corrispondenza:

Dr.ssa Luisa Sternfeld Pavia, AIRP Associazione Italiana Rene Policistico Onlus, Via Bazzini 2, 20I3I Milano.

E-mail: luisa.sternfeld.airp@renepolicistico.it 